\title{
Assessment of Standardization Circumferential Resection Margin Status Influence Survival following Pancreaticoduodenectomy for Pancreatic Ductal Adenocarcinoma
}

Jinheng Liu

West China School of Medicine: Sichuan University West China Hospital

Yanting Wang

Sichuan University West China College of Public Health: Sichuan University West China School of Public Health

Xubao Liu

West China School of Medicine: Sichuan University West China Hospital

Nengwen Ke ( $\nabla$ kenengwen@scu.edu.cn)

West china hospital

\section{Research article}

Keywords: Resection Margin, $1 \mathrm{~mm}$ clearance, Adjuvant chemotherapy, Survival prognosis

Posted Date: December 1st, 2020

DOI: https://doi.org/10.21203/rs.3.rs-113904/v1

License: (9) This work is licensed under a Creative Commons Attribution 4.0 International License.

Read Full License 


\section{Abstract}

Background: Not only to assess the prognostic influence on standardization circumferential resection margin R0 and R1 Status but also to research the prognostic influence on adjuvant chemotherapy to $P V / S M V$, SMA resection margins $₫ 1 \mathrm{~mm}$. The SMV and SMA resection margins had an important prognostic influence to PDAC patients, and the survival prognosis of R1 status(resection margin $₫ 1 \mathrm{~mm}$ ) was poor.

Methods: A total of 228 patients performed PD between 2015 and 2019 were included, which was assessment of standardization circumferential resection margin status and survival prognosis. There were cancer cells within $1 \mathrm{~mm}$ clearance of $\mathrm{PV} / \mathrm{SMV}$ and SMA resection margins named R1 PV/SMV, SMA, and no cancer cells named R0 PV/SMV, SMA.

Results: The resection margin $1 \mathrm{~mm}$ clearance of PV/SMV, SMA $(\mathrm{P}=0.010)$ and postoperative adjuvant chemotherapy $(P=0.001)$ were prognostic independent predictors. The median survival time was 22 months of $166 \mathrm{R} 0$ PV/SMV, SMA patients (73\%) compared to 15 months of $62 \mathrm{R} 1 \mathrm{PV} / \mathrm{SMV}$, SMA patients (27\%) $(P=0.005)$. There was the statistical significance of survival time between the adjuvant chemotherapy group and the none-adjuvant chemotherapy group $(P=0.000)$. In the R1 PV/SMV, SMA group, there was no statistical significance of survival time between the adjuvant chemotherapy patients and the noneadjuvant chemotherapy patients $(P=0.208)$.

Conclusions: Patients undergoing PD for PDAC, postoperative adjuvant chemotherapy could not improve the poor survival prognosis of R1 PV/SMV, SMA resection patients. The resection margins of PV/SMV, SMA had a greater prognostic influence on survival than postoperative adjuvant chemotherapy.

Trial registration: Clinicaltrials.gov/ct2/show/NCT02928081

\section{Background}

Pancreatic ductal adenocarcinoma (PDAC) is the malignant tumor of the digestive system with a poor prognosis. The latest research report showed that the annual incidence of PDAC was 12.9/100000, and the death rate was $11 / 100000^{[1]}$. In the last 20 years, because of progression the comprehensive treatment, the 5-year survival rate of PDAC was higher than before ${ }^{[2,3]}$. Compared to the other malignant tumor, however, the 5 -year survival rate of PDAC was only $8 \%$ and it was still lower ${ }^{[4]}$. The 5 -year survival rate of PDAC patients who had received surgical treatment would reach $12.4 \%$, and the 5 -year survival rate of PDAC patient who was metastatic disease could only be $2.9 \%^{[3,4]}$. And the latest studies predict that the pancreatic adenocarcinoma will be the second most fatal malignancy in $2030^{[5]}$. Nowadays, surgical treatment was still the only radical cure method for PDAC ${ }^{[6-8]}$.

The $90 \%$ of PDAC located in pancreatic head. Previous studies had shown that resection margin was an independent prognostic factor affecting the prognosis of pancreatic head adenocarcinoma ${ }^{[9-15]}$. 
However, some studies had shown that the resection margin was not the independent prognostic factor [16-19]. The reasons caused those results might be none standardized definition of resection margins or none standardized pancreaticoduodenectomy(PD). Then, the Royal College of Pathologists (RCP) had recommended the definition of $\mathrm{R} 1$ status was that there were the cancer cells within the $1 \mathrm{~mm}$ clearance of the circumferential margin of pancreatic surgical specimen ${ }^{[20,21]}$. After that, the International Study Group of Pancreatic Surgery (ISGPS) and College of American Pathologists (CAP) also recommended whether there were cancer cells within the resection margin $1 \mathrm{~mm}$ to define the R0 status or R1 status ${ }^{[22,}$ 23].

With the standard of pathological examination for the pancreatic resection margins, many studies had begun to focus on prognostic influence of pancreatic resection margins $[10,11,14,24]$. Those studies had revealed that in resectable pancreatic head carcinoma patients (exclude vascular invasion patients) who the cancer cells were not microscopic examined within $1 \mathrm{~mm}$ clearance of resection margins, the 5-year survival rate could reach $48 \%$. However, in the patients who the cancer cells were microscopic examined within $1 \mathrm{~mm}$ clearance of resection margins, the 5 -year survival rate was only $14 \%^{[10,11,14,24]}$. Those studies also revealed that the resection margins were an independent risk factor for survival prognosis, and the $\mathrm{R} 1$ resection status had had a poor survival prognosis. And there were multiple resection margins of PD. Whether each resection margin had the same effect on survival prognosis? And, whether it was prognostic significance to study each resection margin? Those questions were controversial. Previous studies had suggested that each resection margin of PDAC had the same effect on survival prognosis ${ }^{[25-29]}$. In 2020, a study in < Pancreatology > had revealed that not all resection margin positivity had the same prognostic significance, and superior mesenteric artery(SMA) and superior mesenteric vein(SMV) margin positivity had a worse survival impact ${ }^{[30]}$.

In the previous studies, the SMV and SMA resection margins had an important prognostic influence to PDAC patients, and the survival prognosis of R1 status(resection margin $₫ 1 \mathrm{~mm}$ ) was poor. However, the previous results had not analyzed the prognostic influence of adjuvant chemotherapy on the R1 status patients. Whether efficacy of adjuvant therapy could improve the survival prognosis of R1 status patients? And whether the R1 status patients who had received adjuvant chemotherapy could be the same survival rate to the R0 status patients? If so, then the patients of resectable pancreatic head carcinoma would perform PD first and then received adjuvant chemotherapy.

In this study, we had examined all the resection margins of PD and assessed the Port Vein/SMV, SMA resection margins. We defined the microscopic examined cancer cells within $1 \mathrm{~mm}$ clearance of PV/SMV, SMA resection margins as $\mathrm{R} 1$ resection(resection margins $\mathbb{1} \mathrm{mm}$ ), and none microscopic examined cancer cells within $1 \mathrm{~mm}$ clearance of PV/SMV, SMA resection margins as R0 resection(resection margins $₫ 1 \mathrm{~mm}$ ). Consequently, we not only assessed the prognostic influence on R0 and R1 status but also researched the prognostic influence on adjuvant chemotherapy to PV/SMV, SMA resection margins $₫ 1 \mathrm{~mm}$. 


\section{Methods \\ Patients}

This study was a retrospective cohort study (Clinicaltrials.gov/ct2/show/NCT02928081), which was approved by the Ethics Committee of Sichuan University West China Hospital (No.2016:122). All patients underwent surgical treatment in the Department of Pancreatic Surgery, Sichuan University West China Hospital, during a 4-year period (January 1, 2015 to March 31, 2019). The decision to perform surgical treatment was made by a multidisciplinary team (MDT) including radiologists, surgeons, pathologists and oncologists. All patients were performed classic PD by 2 surgeons (XB.L and NW.K). This study was limited to patients undergoing PD for resection of pancreatic head adenocarcinoma which all the pathological diagnosis was PDAC. And other lesions such as ampullary, intraductal papillary mucinous neoplasms (IPMN), mucinous cystadenocarcinomas, duodenal or distal bile duct adenocarcinomas and were excluded. And we also had excluded: (a) the patients had received neoadjuvant therapy, (b) the pathological report had not clearly defined the distance between the circumferential margin (CRM) and the tumor.

The inclusion criteria were: (a) age 18-75 years, (b) the computed tomography (CT) showed the tumor had localized in the head of the pancreas, (c) there was no greater than $180^{\circ}$ circumferential involvement of SMV and no overt arterial involvement ${ }^{[14]}$, (d) follow-up data were completed.

No patients got neoadjuvant therapy. Postoperatively, some patients did not choose to receive chemotherapy. Follow-up which was up to December 4, 2019, comprised the out-patient and telephone reviews. When the Carbohydrate antigen19-9 (CA19-9) was high, CT scans were given to the postoperative patients.

\section{Pathology Assessment}

Measurement, stained and fixation of the surgical specimen and definition of the resection margins, pathological examination, and pathological report were all based on the guidelines of the RCPath. (https://www.rcpath.org/profession/guidelines/cancer-data sets-and-tissue-pathways.html)

We had paid particular attention to the CRM definition of the postoperative specimens, which included surface of anterior, posterior, SMV, and SMA (FIGURE 1A). And also, we had examined the transection margins which consisted of the corpus/neck margin, proximal and distal gastric/ jejunum margins and the bile duct margin. Then we had stained the PV/SMV and SMA resection margins(FIGURE 1B), with glacial acetic acid strengthened. The standard of choosing a transverse plane was not only to show the relationship between tumor and adjacent tissues but to show the distance between tumor and resection margin. Thereafter 3 cuts were made from the tumor in head of pancreas. Then the specimens were fixed with $10 \%$ neutral formalin solution for 24 to 48 hours. After that, the specimen was further microscopic 
examined as follows: the tumor, anterior margin, posterior margin, PV/SMV and SMA margin, proximal gastric margin, distal jejunum margin, common bile duct margin and gallbladder, corpus/neck margin [26]

In microscopic assessment, each resection margin was measured at $1 / 10 \mathrm{~mm}$ intervals up to $5 \mathrm{~mm}$ which the blocks of resection margins were cut. And the standard pathological report included as follows: maximum tumor diameter and extent; tumor grade and location of local spread; lymphatic, venous and perineural invasion; total number of lymph nodes examined; number positive lymph nodes. We had performed Tumor Node Metastasis (TNM) which corresponded to the staging system of Union for International Cancer Control (UICC) or American Joint Commission on Cancer (AJCC).

In this study, we had microscopically assessed the R0 or R1 status according to the RCPath criteria. And we had defined the microscopically positive margin as $<1 \mathrm{~mm}$ when there were cancer cells within $1 \mathrm{~mm}$ clearance of PV/SMV and SMA resection margins, and the other margins were not. Those formed the $\mathrm{PV} / \mathrm{SMV}$ and SMA resection margins $>1 \mathrm{~mm}$ group $(\mathrm{R} 1 \mathrm{PV} / \mathrm{SMV}, \mathrm{SMA})$. Also, we had defined the microscopically negative margin as $>1 \mathrm{~mm}$ when there were no cancer cells within $1 \mathrm{~mm}$ clearance of PV/SMV and SMA resection margins, and the other margins were not as well. And those formed the $\mathrm{PV} / \mathrm{SMV}$ and SMA resection margins $>1 \mathrm{~mm}$ group(R0 PV/SMV, SMA $)$.

\section{Statistical Analysis}

All statistical analyses were processed by SPSS version 20.0 (SPSS Inc. Chicago, IL). The $\chi^{2}$ test was used for counting data, and Fisher's exact test was used for frequency which was less than 5 . ShapiroWilk test analyzed the normal distribution of data, and the Levebe test analyzed the homogeneity of variance. The T-test was used for Gaussian distribution, which was expressed as mean \pm standard deviation, and the statistical value was $t$. And the Mann-Whitney $U$ test was used for the abnormal distribution, which was expressed as M (P25, P75), and the statistical value was $Z$. Kaplan-Meier survival analysis analyzed the overall survival withdrawing a survival curve. The Log-Rank test was used for univariate analysis. The proportional hazards regression (Cox regression) model was used for multifactor analysis. Statistical significance was set at a $P<0.05$.

\section{Results}

\section{Different Resection Margin Status of the Patient Cohort from Pathological Report}

Patients whose resection margins were not clearly indicated in the pathological report were excluded. There were a total of 228 patients performed PD between 2015 and 2019 in this study. (FIGURE 2)According to the Pathological report: there were no cancer cells in anterior margin, posterior margin, proximal gastric margin, distal jejunum margin, common bile duct margin and gallbladder, corpus/neck margin of the all the 228 patients. $62(27 \%)$ patients had histologically positive which there were cancer 
cells within $1 \mathrm{~mm}(<1 \mathrm{~mm})$ in PV/SMV and SMA margins and were R1 PV/SMV, SMA resections group. The others $166(73 \%)$ patients which there were no cancer cells within $1 \mathrm{~mm}(>1 \mathrm{~mm})$ were R0 PV/SMV, SMA resections group.

\section{Clinico-pathologic Characteristics Of Different Resection Margin Status Groups}

In this study, tumor grade is categorized into poorly differentiated tumors, poorly-moderately differentiated tumors, moderately differentiated tumors, moderately-well differentiated tumors and well differentiated tumors. All patients were tumor stage T1, T2 or T3. And the AJCC criteria of TNM staging were applied. The clinico-pathologic characteristics of the different resection margin status groups are summarized in Table 1. 
Table 1

Clinico-Pathologic Characteristics of Different Resection Margin Status Groups

\begin{tabular}{|c|c|c|c|c|c|c|}
\hline & & $\begin{array}{l}\text { Total patients } \\
228(100 \%)\end{array}$ & $\begin{array}{l}\mathrm{RO}_{\mathrm{PV} / \mathrm{SMV}, \mathrm{SMA}} \\
166(73 \%)\end{array}$ & $\begin{array}{l}\text { R1 PV/SMV, SMA } \\
62(27 \%)\end{array}$ & $x^{2} / t / Z$ & $P$ \\
\hline Age, year & $\begin{array}{l}M \\
(P 25, P 75)\end{array}$ & $\begin{array}{l}60.5 \\
(53,68.25)\end{array}$ & $\begin{array}{l}61 \\
(53,69)\end{array}$ & $\begin{array}{l}57 \\
(52,66.25)\end{array}$ & -1.467 & 0.142 \\
\hline Gender & & & & & 0.006 & 0.938 \\
\hline Male & $\mathrm{n}(\%)$ & $137(60)$ & $100(60)$ & $37(60)$ & & \\
\hline Female & $\mathrm{n}(\%)$ & $91(40)$ & $66(40)$ & $25(40)$ & & \\
\hline BMI, kg/m² & $\begin{array}{l}M \\
(P 25, P 75)\end{array}$ & $\begin{array}{l}21.495 \\
(19.53,23.83)\end{array}$ & $\begin{array}{l}21.53 \\
(19.38,23.72)\end{array}$ & $\begin{array}{l}21.485 \\
(19.99,23.97)\end{array}$ & -0.661 & 0.509 \\
\hline Diameter, cm & $\begin{array}{l}M \\
\text { (P25,P75) }\end{array}$ & $\begin{array}{l}3 \\
(2.5,3.825)\end{array}$ & $\begin{array}{l}3 \\
(2.475,4)\end{array}$ & $\begin{array}{l}3 \\
(2.5,3.8)\end{array}$ & -0.890 & 0.373 \\
\hline CA19-9, U/ml & $\begin{array}{l}M \\
\text { (P25,P75) }\end{array}$ & $\begin{array}{l}102.75 \\
(16.14,309.35)\end{array}$ & $\begin{array}{l}106.3 \\
(18.83,334.9)\end{array}$ & $\begin{array}{l}83.785 \\
(6.125,271.55)\end{array}$ & -0.605 & 0.545 \\
\hline HGB, g/L & $\begin{array}{l}M \\
\text { (P25,P75) }\end{array}$ & $\begin{array}{l}123.5 \\
(113,133.25)\end{array}$ & $\begin{array}{l}124 \\
(113,135)\end{array}$ & $\begin{array}{l}123 \\
(113,132)\end{array}$ & -0.143 & 0.887 \\
\hline TBIL, $\mu \mathrm{mol} / \mathrm{L}$ & $\begin{array}{l}M \\
(P 25, P 75)\end{array}$ & $\begin{array}{l}106.9 \\
(12.75,223.08)\end{array}$ & $\begin{array}{l}95.4 \\
(12.75,209)\end{array}$ & $\begin{array}{l}139.95 \\
(11.75,232.95)\end{array}$ & -0.818 & 0.413 \\
\hline ALT, U/L & $\begin{array}{l}M \\
(P 25, P 75)\end{array}$ & $86(26,187)$ & $\begin{array}{l}82 \\
(23.5,209)\end{array}$ & $\begin{array}{l}97 \\
(41,160.75)\end{array}$ & -0.407 & 0.684 \\
\hline AST, U/L & $\begin{array}{l}M \\
(P 25, P 75)\end{array}$ & $\begin{array}{l}59 \\
(28,140.5)\end{array}$ & $\begin{array}{l}57.5 \\
(24,142.75)\end{array}$ & $\begin{array}{l}59 \\
(38.75,132.5)\end{array}$ & -0.451 & 0.652 \\
\hline ALB, $g / L$ & $\begin{array}{l}M \\
\text { (P25,P75) }\end{array}$ & $\begin{array}{l}40.05 \\
(37.08,43.43)\end{array}$ & $\begin{array}{l}39.9 \\
(37.13,43.25)\end{array}$ & $\begin{array}{l}40.55 \\
(37.08,43.98)\end{array}$ & -0.690 & 0.490 \\
\hline GLU, mmol/L & $\begin{array}{l}M \\
(P 25, P 75)\end{array}$ & $\begin{array}{l}5.81 \\
(5.09,7.10)\end{array}$ & $\begin{array}{l}5.845 \\
(5.25,7.44)\end{array}$ & $\begin{array}{l}5.65 \\
(4.94,6.71)\end{array}$ & -1.390 & 0.165 \\
\hline $\begin{array}{l}\text { Adjuvant } \\
\text { Chemotherapy }\end{array}$ & & & & & 0.063 & 0.801 \\
\hline Yes & $\mathrm{n}(\%)$ & $95(42)$ & $70(42)$ & $25(40)$ & & \\
\hline
\end{tabular}




\begin{tabular}{|c|c|c|c|c|c|c|}
\hline & & $\begin{array}{l}\text { Total patients } \\
228(100 \%)\end{array}$ & $\begin{array}{l}\mathrm{RO}_{\mathrm{PV} / \mathrm{SMV}, \mathrm{SMA}} \\
166(73 \%)\end{array}$ & $\begin{array}{l}\text { R1 PV/SMV, SMA } \\
62(27 \%)\end{array}$ & $x^{2} / t / Z$ & $P$ \\
\hline No & $\mathrm{n}(\%)$ & 133(58) & $96(58)$ & $37(60)$ & & \\
\hline Grade & & & & & 1.410 & 0.842 \\
\hline poorly & $\mathrm{n}(\%)$ & $28(12)$ & $22(13)$ & $6(10)$ & & \\
\hline $\begin{array}{l}\text { poorly- } \\
\text { moderately }\end{array}$ & $\mathrm{n}(\%)$ & 101(44) & $70(42)$ & $31(50)$ & & \\
\hline moderately & $\mathrm{n}(\%)$ & $81(36)$ & $61(37)$ & $20(32)$ & & \\
\hline moderately-well & $\mathrm{n}(\%)$ & $15(7)$ & $11(7)$ & $4(6)$ & & \\
\hline well & $\mathrm{n}(\%)$ & $3(1)$ & $2(1)$ & $1(2)$ & & \\
\hline $\mathrm{T}$ & & & & & 1.346 & 0.510 \\
\hline 1 & $\mathrm{n}(\%)$ & $40(18)$ & $31(19)$ & $9(15)$ & & \\
\hline 2 & $\mathrm{n}(\%)$ & 153(67) & $112(67)$ & $41(66)$ & & \\
\hline 3 & $\mathrm{n}(\%)$ & $35(15)$ & $23(14)$ & 12(19) & & \\
\hline $\mathbf{N}$ & & & & & 2.645 & 0.448 \\
\hline$x$ & $\mathrm{n}(\%)$ & $42(18)$ & $28(17)$ & $14(23)$ & & \\
\hline 0 & $\mathrm{n}(\%)$ & 108(47) & $84(51)$ & $24(39)$ & & \\
\hline 1 & $\mathrm{n}(\%)$ & $62(28)$ & $43(26)$ & $19(31)$ & & \\
\hline 2 & $\mathrm{n}(\%)$ & $16(7)$ & $11(6)$ & $5(7)$ & & \\
\hline TNM & & & & & 2.923 & 0.571 \\
\hline IA & $\mathrm{n}(\%)$ & $27(12)$ & $22(13)$ & $5(8)$ & & \\
\hline IB & $\mathrm{n}(\%)$ & 71(31) & $53(32)$ & $18(29)$ & & \\
\hline IIA & $\mathrm{n}(\%)$ & $12(5)$ & $10(6)$ & $2(10)$ & & \\
\hline IIB & $\mathrm{n}(\%)$ & $69(31)$ & $48(29)$ & 21(34) & & \\
\hline III & $\mathrm{n}(\%)$ & $49(21)$ & $33(20)$ & 16(19) & & \\
\hline
\end{tabular}


Table 2

A. Result of Log-Rank in Prognostic Factors

\begin{tabular}{|c|c|c|c|c|c|c|}
\hline \multirow[t]{2}{*}{ Characteristics } & \multirow[t]{2}{*}{$\mathbf{N}$} & \multicolumn{3}{|c|}{ Survival Rate\% } & \multirow[t]{2}{*}{$\chi^{2}$} & \multirow[t]{2}{*}{$P$} \\
\hline & & 1-Year & 2-Year & 3-Year & & \\
\hline Resection margin & & & & & 7.871 & 0.005 \\
\hline $\mathrm{R} 0_{\mathrm{PV} / \mathrm{SMV}, \mathrm{SMA}}$ & 166 & $73 \%$ & $37 \%$ & $25 \%$ & & \\
\hline $\mathrm{R} 1_{\mathrm{PV} / \mathrm{SMV}, \mathrm{SMA}}$ & 62 & $61 \%$ & $13 \%$ & $10 \%$ & & \\
\hline Adjuvant Chemotherapy & & & & & 12.759 & 0.000 \\
\hline Yes & 95 & $83 \%$ & $56 \%$ & $31 \%$ & & \\
\hline No & 133 & $65 \%$ & $30 \%$ & $14 \%$ & & \\
\hline Gender & & & & & 0.607 & 0.436 \\
\hline Male & 137 & $72 \%$ & $44 \%$ & $20 \%$ & & \\
\hline Female & 91 & $75 \%$ & $44 \%$ & $20 \%$ & & \\
\hline Age & & & & & 0.418 & 0.518 \\
\hline$\leq 60$ year & 111 & $74 \%$ & $31 \%$ & $24 \%$ & & \\
\hline$\$ 60$ year & 117 & $71 \%$ & $41 \%$ & $16 \%$ & & \\
\hline BMI & & & & & 0.376 & 0.540 \\
\hline Normal & 139 & $74 \%$ & $36 \%$ & $18 \%$ & & \\
\hline Abnormal & 89 & $68 \%$ & $32 \%$ & $20 \%$ & & \\
\hline CA19-9 & & & & & 1.159 & 0.282 \\
\hline Normal & 59 & $71 \%$ & $43 \%$ & $25 \%$ & & \\
\hline Abnormal & 169 & $69 \%$ & $39 \%$ & $19 \%$ & & \\
\hline HGB & & & & & 1.407 & 0.236 \\
\hline Normal & 183 & $74 \%$ & $38 \%$ & $23 \%$ & & \\
\hline Anemia & 45 & $69 \%$ & $21 \%$ & $8 \%$ & & \\
\hline TBIL & & & & & 0.125 & 0.724 \\
\hline Normal & 82 & $75 \%$ & $38 \%$ & $19 \%$ & & \\
\hline Jaundice & 146 & $68 \%$ & $35 \%$ & $17 \%$ & & \\
\hline ALT & & & & & 0.636 & 0.425 \\
\hline
\end{tabular}




\begin{tabular}{|c|c|c|c|c|c|c|}
\hline \multirow[t]{2}{*}{ Characteristics } & \multirow[t]{2}{*}{$\mathbf{N}$} & \multicolumn{3}{|c|}{ Survival Rate\% } & \multirow{2}{*}{$\chi^{2}$} & \multirow[t]{2}{*}{$P$} \\
\hline & & 1-Year & 2-Year & 3-Year & & \\
\hline Normal & 82 & $71 \%$ & $37 \%$ & $23 \%$ & & \\
\hline Abnormal & 146 & $68 \%$ & $30 \%$ & $10 \%$ & & \\
\hline AST & & & & & 2.247 & 0.134 \\
\hline Normal & 73 & $77 \%$ & $38 \%$ & $26 \%$ & & \\
\hline Abnormal & 155 & $71 \%$ & $33 \%$ & $15 \%$ & & \\
\hline ALB & & & & & 1.239 & 0.266 \\
\hline Normal & 194 & $71 \%$ & $36 \%$ & $21 \%$ & & \\
\hline Hypoalbuminemia & 34 & $64 \%$ & $26 \%$ & $14 \%$ & & \\
\hline GLU & & & & & 1.375 & 0.241 \\
\hline Normal & 168 & $71 \%$ & $36 \%$ & $22 \%$ & & \\
\hline Hyperglycemia & 60 & $70 \%$ & $26 \%$ & $15 \%$ & & \\
\hline \multicolumn{2}{|c|}{ Maximum Diameter of Tumor } & & & & 1.364 & 0.505 \\
\hline$\leq 2 \mathrm{~cm}$ & 98 & $76 \%$ & $39 \%$ & $19 \%$ & & \\
\hline$>2 \mathrm{~cm}, \leq 4 \mathrm{~cm}$ & 81 & $70 \%$ & $38 \%$ & $18 \%$ & & \\
\hline$>4 \mathrm{~cm}$ & 49 & $68 \%$ & $32 \%$ & $16 \%$ & & \\
\hline Grade & & & & & 4.199 & 0.380 \\
\hline poorly & 28 & $70 \%$ & $41 \%$ & 0 & & \\
\hline poorly-moderately & 101 & $65 \%$ & $31 \%$ & $16 \%$ & & \\
\hline moderately & 81 & $79 \%$ & $39 \%$ & $17 \%$ & & \\
\hline moderately-well & 15 & $79 \%$ & $63 \%$ & $47 \%$ & & \\
\hline well & 3 & $99 \%$ & 0 & 0 & & \\
\hline TNM & & & & & 3.064 & 0.547 \\
\hline IA & 27 & $80 \%$ & $46 \%$ & $25 \%$ & & \\
\hline IB & 71 & $75 \%$ & $43 \%$ & $7 \%$ & & \\
\hline IIA & 12 & $61 \%$ & $61 \%$ & $30 \%$ & & \\
\hline IIB & 69 & $64 \%$ & $32 \%$ & $15 \%$ & & \\
\hline III & 49 & $68 \%$ & $21 \%$ & $17 \%$ & & \\
\hline
\end{tabular}




\begin{tabular}{|c|c|c|c|c|c|c|}
\hline \multirow[t]{2}{*}{ Characteristics } & \multirow[t]{2}{*}{$\mathbf{N}$} & \multicolumn{3}{|c|}{ Survival Rate\% } & \multirow{2}{*}{$x^{2}$} & \multirow[t]{2}{*}{$P$} \\
\hline & & 1-Year & 2-Year & 3-Year & & \\
\hline \multicolumn{7}{|c|}{ Table 2B. Result of Multivariate Analysis in Prognostic Factors } \\
\hline Characteristics & HR & 95\%C.I & & & & $\mathrm{P}$ \\
\hline Resection Margin & 1.649 & 1.126 & & 2.415 & & 0.010 \\
\hline Adjuvant Chemotherapy & 1.896 & 1.303 & & 2.758 & & 0.001 \\
\hline
\end{tabular}

\section{Survival Prognostic Factors By Univariate And Multivariate Analysis}

The results of the Log-rank test showed that resection margins status $(P=0.005)$ and adjuvant chemotherapy $(P=0.000)$ were associated with survival prognosis (Table $2 A)$. The results of Cox regression showed that resection margins status $(P=0.010)$ and adjuvant chemotherapy $(P=0.001)$ were independent risk factors for survival prognosis (Table 2B).

\section{Relationship Between Survival And Resection Margin Status}

In a total of 228 patients, 215(94.3\%) patients were followed up after surgical. 152(91.6\%) patients were followed up in the R0 PV/SMV, SMA group. And 57(91.9\%) patients were followed up in the R1 PV/SMV, SMA group. The follow-up time ranged 6-60 months, and the median follow-up time was 20 months (Table 3 ) 
Table 3

Survival Analysis between Resection Margin Status and Adjuvant Chemotherapy

\begin{tabular}{|c|c|c|c|c|c|c|c|}
\hline & $\begin{array}{l}\text { Survival } \\
\text { time (M) }\end{array}$ & $\begin{array}{l}\text { Median survival } \\
\text { time (M) }\end{array}$ & $\begin{array}{l}\text { Mean survival } \\
\text { time (M) }\end{array}$ & $\begin{array}{l}1- \\
\text { Year }\end{array}$ & $\begin{array}{l}2- \\
\text { Year }\end{array}$ & $\begin{array}{l}\text { 3- } \\
\text { Year }\end{array}$ & $P$ \\
\hline $\begin{array}{l}\text { Total } \\
\text { patients }\end{array}$ & $0.3-52$ & 21 & $15.75 \pm 8.90$ & $73 \%$ & $40 \%$ & $19 \%$ & - \\
\hline \multicolumn{8}{|l|}{$\begin{array}{l}228 \\
(100 \%)\end{array}$} \\
\hline \multicolumn{8}{|c|}{ Resection margin (228) } \\
\hline $\begin{array}{l}\mathrm{RO}_{\mathrm{PV} / \mathrm{SMV}} \\
\mathrm{SMA}\end{array}$ & $0.3-52$ & 22 & $16.98 \pm 8.99$ & $73 \%$ & $37 \%$ & $25 \%$ & 0.005 \\
\hline $166(73 \%)$ & & & & & & & \\
\hline $\begin{array}{l}\text { R1 PV/SMV, } \\
\text { SMA }\end{array}$ & $0.6-42$ & 15 & $13.22 \pm 8.21$ & $61 \%$ & $13 \%$ & $10 \%$ & \\
\hline $62(27 \%)$ & & & & & & & \\
\hline \multicolumn{8}{|c|}{ Adjuvant chemotherapy (228) } \\
\hline $\begin{array}{l}\text { Yes } \\
95(42 \%)\end{array}$ & $6-43$ & 24 & $17.28 \pm 8.48$ & $83 \%$ & $56 \%$ & $31 \%$ & 0.000 \\
\hline $\begin{array}{l}\text { No } \\
133(58 \%)\end{array}$ & $0.3-52$ & 17 & $14.59 \pm 9.08$ & $65 \%$ & $30 \%$ & $14 \%$ & \\
\hline \multicolumn{8}{|c|}{ None-adjuvant chemotherapy group (133) } \\
\hline $\begin{array}{l}\mathrm{RO}_{\mathrm{PV} / \mathrm{SMV}} \\
\mathrm{SMA}\end{array}$ & $0.3-52$ & 19 & $15.7 \pm 9.53$ & $69 \%$ & $36 \%$ & $15 \%$ & 0.074 \\
\hline $96(72 \%)$ & & & & & & & \\
\hline $\begin{array}{l}\text { R1 PV/SMV, } \\
\text { SMA }\end{array}$ & $0.6-35$ & 15 & $12.66 \pm 8.00$ & $54 \%$ & $11 \%$ & $0 \%$ & \\
\hline $37(28 \%)$ & & & & & & & \\
\hline \multicolumn{8}{|c|}{ Adjuvant chemotherapy group (95) } \\
\hline $\begin{array}{l}\mathrm{RO}_{\mathrm{PV} / \mathrm{SMV}} \\
\text { SMA } \\
70(74 \%)\end{array}$ & $6-43$ & 26 & $18.42 \pm 8.22$ & $84 \%$ & $63 \%$ & $35 \%$ & 0.025 \\
\hline $\begin{array}{l}\text { R1 PV/SMV, } \\
\text { SMA } \\
25(26 \%)\end{array}$ & $2-42$ & 16 & $14.11 \pm 8.61$ & $79 \%$ & $25 \%$ & $13 \%$ & \\
\hline
\end{tabular}




\begin{tabular}{|c|c|c|c|c|c|c|c|}
\hline & $\begin{array}{l}\text { Survival } \\
\text { time (M) }\end{array}$ & $\begin{array}{l}\text { Median survival } \\
\text { time (M) }\end{array}$ & $\begin{array}{l}\text { Mean survival } \\
\text { time (M) }\end{array}$ & $\begin{array}{l}1- \\
\text { Year }\end{array}$ & $\begin{array}{l}2- \\
\text { Year }\end{array}$ & $\begin{array}{l}3- \\
\text { Year }\end{array}$ & $P$ \\
\hline \multicolumn{8}{|c|}{ The Relationship between R0 $0_{\mathrm{PV} / \mathrm{SMV} \text {, SMA }}$ Patients and Adjuvant chemotherapy (166) } \\
\hline \multicolumn{8}{|c|}{ Adjuvant chemotherapy } \\
\hline $\begin{array}{l}\text { Yes } \\
70(42 \%)\end{array}$ & $6-43$ & 26 & $18.42 \pm 8.22$ & $84 \%$ & $63 \%$ & $35 \%$ & \multirow[t]{2}{*}{0.001} \\
\hline $\begin{array}{l}\text { No } \\
96(58 \%)\end{array}$ & $0.3-52$ & 19 & $15.7 \pm 9.53$ & $69 \%$ & $36 \%$ & $15 \%$ & \\
\hline \multicolumn{8}{|c|}{ The Relationship between R1 PV/SMV, SMA Patients and Adjuvant chemotherapy (62) } \\
\hline \multicolumn{8}{|c|}{ Adjuvant chemotherapy } \\
\hline $\begin{array}{l}\text { Yes } \\
25(40 \%)\end{array}$ & $2-42$ & 16 & $14.11 \pm 8.61$ & $79 \%$ & $25 \%$ & $13 \%$ & \multirow[t]{2}{*}{0.208} \\
\hline $\begin{array}{l}\text { No } \\
37(60 \%)\end{array}$ & $0.6-35$ & 15 & $12.66 \pm 8.00$ & $54 \%$ & $11 \%$ & $0 \%$ & \\
\hline
\end{tabular}

In a total of 228 patients, the survival time ranged $0.3-52$ months, the median survival time was 21 months, the mean survival time was $15.75 \pm 8.90$ months and the 1-year, 2-year, and 3-year survival rates were $73 \%, 40 \%$, and $19 \%$, respectively. In the R0 PV/SMV, SMA group, the survival time ranged $0.3-52$ months, the median survival time was 22 months, the mean survival time was $16.98 \pm 8.99$ months and the 1-year, 2-year, and 3-year survival rates were $73 \%, 37 \%$, and $25 \%$, respectively. In the R1 PV/SMV, SMA group, the survival time ranged 0.6-42 months, the median survival time was 15 months, the mean survival time was $13.22 \pm 8.21$ months, and the 1-year, 2-year, and 3-year survival rates were $61 \%, 13 \%$, and $10 \%$, respectively. There was statistical significance of survival time between R1 PV/SMV, SMA group and R0 PV/SMV, SMA group $(P=0.005)$ (Table 3)(FIGURE 3 A).

\section{Survival Relationship Between Adjuvant Chemotherapy And Resection Margin Status}

Postoperatively, 95 (42\%) patients had received adjuvant chemotherapy. 24 patients were treated with Gemcitabine, 57 patients were treated with Tegafur Gimeracil Oteracil Potassium Capsule, 4 patients were treated with Tegafur Gimeracil Oteracil Potassium Capsule combined Gemcitabine. 4 patients were treated with FOLFIRINOX and 6 with Doxifluridine. In addition to this, $133(58 \%)$ patients had not received any adjuvant therapy.

There was the statistical significance of survival time between the adjuvant chemotherapy group and the none-adjuvant chemotherapy group ( $P=0.000$ ), (Table 3)(FIGURE 3B). In the none-adjuvant chemotherapy group, there was no statistical significance of survival time between R0 PV/SMV, SMA patients and R1 PV/SMV, SMA patients( $P=0.074$ ) (Table 3) (FIGURE 3C). In the adjuvant chemotherapy 
group, there was statistical significance of survival time between R0 PV/SMV, SMA group and R1 PV/SMV, SMA group $(P=0.025)$. (Table 3) (FIGURE 3D)

In the R0 PV/SMV, SMA group, there was the statistical significance of survival time between the adjuvant chemotherapy patients and the none-adjuvant chemotherapy patients $(P=0.001)$ (Table 3 ) (FIGURE 3E). In the R1 PV/SMV, SMA group, there was no statistical significance of survival time between the adjuvant chemotherapy patients and the none-adjuvant chemotherapy patients $(P=0.208)$ (Table 3$)$ (FIGURE 3F).

\section{Discussion}

The previous study had suggested that the patients of $\mathrm{R} 1_{\mathrm{PV} / \mathrm{SMV}}$, SMA resection had a poor survival prognosis. And in this study, resection margin $(P=0.010)$ and postoperative adjuvant chemotherapy ( $P=$ 0.001 ) were independent risk factors of survival prognosis. We had researched the survival prognosis of $\mathrm{PV} / \mathrm{SMV}$ and SMA resection margins. Except the PV/SMV and SMA resection margins, the other resection margins in PD were R0 status according to the $1 \mathrm{~mm}$ clearance principle of RCPath. For many of the previous studies had failed to demonstrate that adjuvant chemotherapy influenced the outcome of R1 status. And specific details of relationship between the PV/SMV and SMA resection margins and the adjuvant chemotherapy were lacking in previous studies. Then, we had not only analyzed the survival prognosis of R1 PV/SMV, SMA status but analyzed whether the adjuvant chemotherapy could improve the survival prognosis of R1 PV/SMV, SMA status.

Previous studies had revealed that smoking and obesity were modifiable risk factors for PDAC, and that was to say, smoking cessation and diet could reduce the risk of PDAC ${ }^{[31]}$. Male had nearly $30 \%$ more than women for the incidence of PDAC ${ }^{[32]}$. In our study, 137 (60\%) patients were male, and 91 (40\%) patients were female, which was consistent with previous studies. The cause of difference in incidence might be male's preference for alcohol, smoking and obesity. However, the gender, age and BMI were not the independent prognostic factors in this study, which might relate to the small sample and PDAC had caused patients to lose weight quickly.

A series of previous studies on resection margins in which the standardized histopathological examination had reported that the resection margins had prognostic influence on survival. Popescu I et al had reported that RO resection of PD in PDAC could improve overall postoperative survival rate ${ }^{[33]}$. Tummers W.S et al had researched the relationship between the R0 and (or) R1 resection and postoperative local recurrence, overall survival, which had reported that the $\mathrm{R}$ status of resection margin was associated with the postoperative local recurrence, overall survival of PDAC patients ${ }^{[34]}$. Especially in N1staging patients, there were having similar recurrence characteristics between R0 and R1 resection ${ }^{[34]}$. Demir I.E et al had reported that the PD for advanced PDAC was more likely to be R1 resection, and the resection margins could be independent risk factor for survival prognosis ${ }^{[35]}$. In a prospective randomized controlled study, Delpero J.R et a/had also reported that the $\mathrm{R}$ status of resection margin was associated with the survival prognosis, and they further reported that resection margins $<1 \mathrm{~mm}$ was 
independent risk factor for survival prognosis ${ }^{[36]}$. After standardized histopathological examination on the resection margins of specimen, Hank $\mathrm{T}$ et al had reported that the resection margins $1 \mathrm{~mm}$ was independent risk factor for survival prognosis ${ }^{[37]}$. In this study, there was statistical significance between $\mathrm{R} 1 \mathrm{PV} / \mathrm{SMV}$, SMA group and R0 PV/SMV, SMA group $(P=0.005)$. And the 1, 2, 3-year survival rate of R0 PV/SMV, SMA group patients were significantly better than that of R1 PV/SMV, SMA group(73\%VS 61\% $₫ 40 \%$ VS 13\%》 $19 \%$ VS 10\%). Therefore, the resection margin $1 \mathrm{~mm}$ clearance of PV/SMV, SMA was the independent risk factor for survival prognosis in this study.

With the standardized histopathological examination on the specimen, most R0 resection of PD in the past was actually $\mathrm{R} 1$ resection, which the reason might be that the PV/SMV, SMA resection margins were nonstandardized of radical resection. For this cohort of patients, neoadjuvant therapy and adjuvant chemotherapy might have been critical to improving survival prognosis. NCCN had recommended adjuvant chemotherapy following resection of PDAC as standard treatment since 2000. Postoperatively, however, less than $50 \%$ of patients received adjuvant chemotherapy ${ }^{[38]}$. In a retrospective study of PDAC,

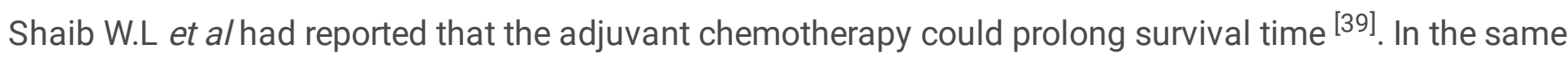
study, Nagrial A.M et a/had reported that the elder PDAC patients who had received postoperative adjuvant chemotherapy could live longer ${ }^{[40]}$. This study had provided adjuvant chemotherapy evidence for elderly PDAC patients after PD. In a study of adjuvant chemotherapy with Gemcitabine, Oettle $\mathrm{H}$ et al had reported that the PDAC patients who had received standardized treatment of Gemcitabine for 6 months would live longer and get higher disease-free survival ${ }^{[41]}$. Then in the study of system evaluation in PDAC patients, Parmar A et a/ had not only confirmed that postoperative adjuvant chemotherapy could significantly improve the survival prognosis but also had recommended the mFOLFIRINOX as the preferred adjuvant chemotherapy regimen ${ }^{[42]}$. And in our study, there was statistical significance of survival time between the adjuvant chemotherapy group and the none-adjuvant chemotherapy group $(P=0.000)$. The adjuvant chemotherapy group patients had higher 1-year, 2-year, and 3-year survival rates than the none-adjuvant chemotherapy group ( $83 \%$ vs $65 \%, 56 \%$ vs $30 \%, 31 \%$ vs $14 \%$ ), which was consistent with previous studies.

When we had analyzed the survival time of subgrouping of the R0 PV/SMV, SMA group, and R1 PV/SMV, SMA group separately by comparison of adjuvant chemotherapy and none- adjuvant chemotherapy. In R0 PV/SMV, SMA group, and adjuvant chemotherapy patients had a longer mean survival time $(18.42 \pm 8.22 \mathrm{~m}$ $v s 15.7 \pm 9.53 \mathrm{~m})$ and a higher 1-year, 2-year, and 3-year survival rates(86\%vs68\% $863 \%$ vs $36 \% \$ 35 \%$ vs $15 \%)$ than none-adjuvant chemotherapy patients. However, in R1 PV/SMV, SMA group, though the adjuvant chemotherapy patients had a higher 1-year, 2-year, and 3-year survival rates than none-adjuvant chemotherapy patients $(75 \% \mathrm{vs} 54 \% \varangle 25 \% \mathrm{vs} 11 \% \varangle 35 \% \mathrm{vs} 0 \%)$, there was no statistical significance in survival analysis between the adjuvant chemotherapy patients and none-adjuvant chemotherapy patients $(P=$ 0.208). In this study, if the R1 $1_{P V / S M V}$, SMA patients would not receive adjuvant chemotherapy, and the 3year survival rate was 0 . And the postoperatively adjuvant chemotherapy could not improve survival 
prognosis of R1 PV/SMV, SMA patients which survival prognosis was very poor. To this cohort of patients, it was insignificance to perform surgical treatment.

\section{Conclusion}

In conclusion, we had demonstrated that in this cohort of 228 patients undergoing PD for PDAC, the $\mathrm{RO}_{\mathrm{PV} / \mathrm{SMV}}$, SMA rate was $73 \%$ and the $\mathrm{R} 1 \mathrm{PV} / \mathrm{SMV}$, SMA rate was $27 \%$, and the resection margin $1 \mathrm{~mm}$ clearance of PV/SMV, SMA was prognostic independent predictor. And the $\mathrm{R} 0_{\mathrm{PV} / \mathrm{SMV}}$, SMA patients had a better survival prognosis. Furthermore, postoperative adjuvant chemotherapy was also prognostic independent predictor in this cohort of 228 patients. However, postoperative adjuvant chemotherapy could not improve the poor survival prognosis of R1 PV/SMV, SMA patients. This result had suggested that the resection margins of PV/SMV, SMA had a greater prognostic influence on survival than postoperative adjuvant chemotherapy. This was small sample and single-center research but should future validation in big data and multiple centers study. It is possible to improve prognostication and efficacy of R1 PV/SMV, SMA patients by early treatment intervention such as neoadjuvant therapy.

\section{Abbreviations}

PDAC

Pancreatic ductal adenocarcinoma

PD

Pancreaticoduodenectomy

RCP

Royal College of Pathologists

ISGPS

International Study Group of Pancreatic Surgery

CAP

College of American Pathologists

SMA

Superior Mesenteric Artery

SMV

Superior Mesenteric Vein

PV

Port Vein

MDT

Multidisciplinary Team

IPMN

Intraductal Papillary Mucinous Neoplasms

CRM

Circumferential Margin 
CT

Computed Tomography

CA19-9

Carbohydrate antigen19-9

TNM

Tumor Node Metastasis

UICC

Union for International Cancer Control

AJCC

American Joint Commission on Cancer

\section{Declarations}

\section{Ethics approval and consent to participate}

This study was a prospective cohort study (Clinicaltrials.gov/ct2/show/NCT02928081), which was approved by the Ethics Committee of Sichuan University West China Hospital (No.2016:122).

\section{Consent for publication}

Consent for publication had be obtained from all patients.

\section{Competing interests}

The authors declare that they have no competing interests.

\section{Funding}

1, NWK had got the support of Scientific and Technological Support Program of Sichuan Province (2020YFS0262).

\section{Authors' contributions}

JHL and YTW contributed equally to this study.

\section{Acknowledgments}


The authors thanked the pancreas multi-disciplinary team members for their continuous support. And thanks Professor Jiajun Qiu for providing purely technical help.

\section{References}

1. US Preventive Services Task Force. Owens DK, Davidson KW. et, al. Screening for Pancreatic Cancer: US Preventive Services Task Force Reaffirmation Recommendation Statement[J]. JAMA. 2019;322(5):438-44. DOI:10.1001/jama.2019.10232.

2. Gudjonsson B. Pancreatic cancer: survival, errors and evidence[J]. European Journal of Gastroenterology Hepatology. 2009;21(12):1379-82. DOI:10.1097/MEG.0b013e328323aab7.

3. Rebecca L. Siegel; Kimberly D, Miller. Ahmedin Jemal; Cancer Statistics, 2019. CA Cancer J Clin. 2019 Jan;69(1):7-34.

4. National Cancer Institute $(\mathrm{NCl})$. Cancer Stat Facts: pancreatic cancer. $\mathrm{NCl}$ website https://seer.cancer.gov/statfacts/html/pancreas. html. Accessed June 12, 2019.

5. Rahib L, Smith BD, Aizenberg R, Rosenzweig AB, Fleshman JM, Matrisian LM. Projecting cancer incidence and deaths to 2030: the unexpected burden of thyroid, liver, and pancreacancers in the United States. Cancer Res. 2014;74:2913-21. DOI:10.1158/0008-5472.CAN-14-0155.

6. Audrey Vincent J, Herman R, Schulick, et al. Pancreatic cancer Lancet. 2011;13(9791):607-20. DOI:10.1016/S0140-6736(10)62307-0. 378 ) .

7. Hartwig W, Werner J, Ja“ger D, et al. Improvement of surgical results for pancreatic cancer. Lancet Oncol. 2013;14:e476-85. DOI:10.1016/S1470-2045(13)70172-4.

8. Maitra A, Hruban RH. Pancreatic Cancer[J]. Annual Review of Pathology Mechanisms of Disease. 2008;3(1):157-88. DOI:10.1146/annurev.pathol.3.121806.154305.

9. Neoptolemos JP, Stocken DD, Friess $\mathrm{H}$, et al. A randomized trial of chemoradiotherapy and chemotherapy after resection of pancreatic cancer. N Engl J Med. 2004;350:1200-10.

DOI:10.1056/NEJMoa032295.

10. Strobel O, Hank T, Hinz U. MSc, et al. Pancreatic Cancer Surgery: The New R-status Counts. Ann Surg. 2017;265:565-73. DOI:10.1097/SLA.0000000000001731.

11. Demir IE, Jäger C, Schlitter AM, Konukiewitz B, Stecher L, Schorn S, Tieftrunk E, Scheufele F, Calavrezos L, Schirren R, Esposito I, Weichert W, Friess H, Ceyhan GO. R0 Versus R1 Resection Matters after Pancreaticoduodenectomy, and Less after Distal or Total Pancreatectomy for Pancreatic Cancer. Annals of Surgery. 2018 Dec;268(6):1058-1068. DOI: $10.1097 /$ SLA.0000000000002345.

12. Yamamoto $\mathrm{T}$, Uchida $\mathrm{Y}$, Terajima $\mathrm{H}$. Clinical impact of margin status on survival and recurrence pattern after curative-intent surgery for pancreatic cancer. Asian J Surg. 2019 Jan;42(1):93-9. DOI:10.1016/j.asjsur.2017.09.003.

13. Neoptolemos JP, Stocken DD, Dunn JA, et al. Influence of resection margins on survival for patients with pancreatic cancer treated by adjuvant chemoradiation and/or chemotherapy in the ESPAC-1 
randomized controlled trial. Ann Surg. 2001;234:758-68. DOI:10.1097/00000658-200112000-00007.

14. Delpero J, Robert MD, Jeune PhD, Bachellier F, Philippe,et al. Prognostic Value of Resection Margin Involvement After Pancreaticoduodenectomy for Ductal Adenocarcinoma: Updates From a French Prospective Multicenter Study. Ann Surg. 2017;266(5):787-96.

DOI:10.1097/SLA.0000000000002432.

15. Westgaard A, Tafjord S, Farstad IN, et al. Resectable adenocarcinomas in the pancreatic head: the retroperitoneal resection margin is an independent prognostic factor. BMC Cancer. 2008;8:5.

DOI:10.1186/1471-2407-8-5.

16. Raut CP, Tseng JF, Sun CC, Wang H, Wolff RA, Crane CH, et al. Impact of resection status on pattern of failure and survival after pancreaticoduodenectomy for pancreatic adenocarcinoma. Ann Surg. 2007;246:52-60. DOI:10.1097/01.sla.0000259391.84304.2b.

17. Neoptolemos JP, Stocken DD, Bassi C, Ghaneh P, Cunningham D, Goldstein D, et al.; European Study Group for Pancreatic Cancer. Adjuvant chemotherapy with fluorouracil plus folinic acid vs gemcitabine following pancreatic cancer resection: a randomized controlled trial.JAMA 2010; 304: 1073-1081. DOI: 10.1001/jama.2010.1275.

18. Neoptolemos JP, Stocken DD, Friess H, Bassi C, Dunn JA, Hickey H, et al. European Study Group for Pancreatic Cancer. A randomized trial of chemoradiotherapy and chemotherapy after resection of pancreatic cancer. N Engl J Med. 2004;350:1200-10. DOI:10.1056/NEJMoa032295.

19. Kato K, Yamada S, Sugimoto H, Kanazumi N, Nomoto S, Takeda S, et al. Prognostic factors for survival after extended pancreatectomy for pancreatic head cancer: influence of resection margin status on survival. Pancreas. 2009;38:605-12. DOI:10.1097/MPA.0b013e3181a4891d.

20. Gill AJ. J. Synoptic reporting improves histopathological assessment of pancreatic resection specimens[J]. Pathology. 2009;41:161-7. DOI:10.1080/00313020802337329.

21. The Royal College of Pathologists. Standards and minimum datasets for reporting cancers: dataset for the histopathological reporting of carcinomas of the pancreas, ampulla of Vater and common bile duct. London: The Royal College of Pathologists; 2010.

22. Bockhorn M, Uzunoglu FG, Adham M, et al. Borderline resectable pancreatic cancer: A consensus statement by the International Study Group of Pancreatic Surgery (ISGPS). Surgery. 2014;155(6):977-88. DOl:10.1016/j.surg.2014.02.001.

23. Washington K, Berlin J, Branton P, et al. Protocol for the Examination of Specimens from Patients with Carcinoma of the Exocrine Pancreas. Northfield: College of American Pathologists; 2017.

24. Konstantinidis IT, WarshawAL, Allen JN, et al. Pancreatic ductal adenocarcinoma: is there a survival difference for R1 resections versus locally advanced unresectable tumors? What is a 'True' R0 resection? Ann Surg. 2013;257:731-6. DOI:10.1097/SLA.0b013e318263da2f.

25. Ghaneh P, Kleeff J, Halloran CM, et al. The Impact of Positive Resection Margins on Survival and Recurrence Following Resection and Adjuvant Chemotherapy for Pancreatic Ductal Adenocarcinoma[J]. Annals of Surgery, 2017:1. DOI: 10.1097/SLA.0000000000002557. 
26. Verbeke CS, Menon KV.. Redefining resection margin status in pancreatic cancer[J]. Hpb, 2009, 11. DOI:10.1111/j.1477-2574.2009.00055.x.

27. Esposito I, Kleeff J, Bergmann F, et al. Most Pancreatic Cancer Resections are R1 Resections[J]. Ann Surg Oncol. 2008;15(6):1651-60. DOI:10.1245/s10434-008-9839-8.

28. Wagner $\mathrm{M}$, Redaelli $\mathrm{C}$, Lietz $\mathrm{M}$, et al. Curative resection is the single most important factor determining outcome in patients with pancreatic adenocarcinoma.[J]. Br J Surg. 2010;91(5):586-94. DOI:10.1002/bjs.4484.

29. Kuhlmann K, Castro SD, Heek TV, et al. Microscopically incomplete resection offers acceptable palliation in pancreatic cancer[J]. Surgery (St Louis). 2006;139(2):188-96. DOI:10.1002/bjs.4484.

30. Pine JK, Haugk B, Robinson SM, Darne A, Wilson C, Sen G, French JJ, White SA, Manas DM, Charnley $\mathrm{RM}$, Prospective assessment of resection margin status following pancreaticoduodenectomy for pancreatic ductal adenocarcinoma after standardisation of margin definitions, Pancreatology (2020) DOI: 10.1016/j.pan.2020.01.004.

31. Lauby-Secretan B, Scoccianti C, Loomis D, et al. Body Fatness and Cancer - Viewpoint of the IARC Working Group[J]. N Engl J Med. 2016;375(8):794-8. DOI:10.1056/NEJMsr1606602.

32. Motojima $\mathrm{K}$, Tomioka $\mathrm{T}$, Kohara $\mathrm{N}$, et al. Immunohistochemical characteristics of adenosquamous carcinoma of the pancreas[J]. Journal of surgical oncology. 1992;49(1):58-62. DOI:10.1002/jso.2930490114.

33. Popescu I, Dumitrascu T. Total meso-pancreas excision: key point of resection in pancreatic head adenocarcinoma.[J]. 2011, 58(105):202-207. DOI:10.1136/gut.2008.170811corr1.

34. Tummers WS, Groen JV, Sibinga Mulder BG, et al. Impact of resection margin status on recurrence and survival in pancreatic cancer surgery[J]. Br J Surg. 2019;106(8):1055-65.

DOI:10.1002/bjs.11115.

35. Demir IE, Carsten Jäger, Schlitter AM, et al. R0 Versus R1 Resection Matters after Pancreaticoduodenectomy, and Less after Distal or Total Pancreatectomy for Pancreatic Cancer[J]. Ann Surg. 2017;268(6):1. DOI:10.1097/SLA.0000000000002345.

36. Delpero JR, Jeune F, Bachellier P, et al. Prognostic Value of Resection Margin Involvement After Pancreaticoduodenectomy for Ductal Adenocarcinoma: Updates From a French Prospective Multicenter Study[J]. Ann Surg. 2017;266(5):1. DOI:10.1097/SLA.0000000000002432.

37. Validation of at least. $1 \mathrm{~mm}$ as cut-off for resection margins for pancreatic adenocarcinoma of the body and tail[J]. Br J Surg, 2018, 105. DOI:10.1002/bjs.10842.

38. Prognostic Factors of Survival After Neoadjuvant Treatment and Resection for Initially Unresectable Pancreatic. Cancer[J] Annals of Surgery. 2019. DOI:10.1097/SLA.0000000000003270.

39. Role of adjuvant therapy. in resected stage IA subcentimeter (T1a/T1b) pancreatic cancer.[J]. Cancer. 2018. DOI:10.1002/cncr.31787.

40. Adjuvant chemotherapy in. elderly patients with pancreatic cancer[J]. Br J Cancer. 2014;110(2):3139. DOI:10.1038/bjc.2013.722. 
41. Oettle $\mathrm{H}$, Neuhaus $\mathrm{P}$, Hochhaus A, et al. Adjuvant Chemotherapy With Gemcitabine and Long-term Outcomes Among Patients With Resected Pancreatic Cancer The CONKO-001 Randomized Trial[J]. JAMA The Journal of the American Medical Association. 2013;310(14):1473-81. DOI:10.1001/jama.2013.279201.

42. Parmar A, Chaves-Porras J, Saluja R, et al. Adjuvant treatment for resected pancreatic adenocarcinoma: A systematic review and network meta-analysis [J]. Crit Rev Oncol Hematol. 2020;145:102817. DOI:10.1016/j.critrevonc.2019.102817.

\section{Figures}
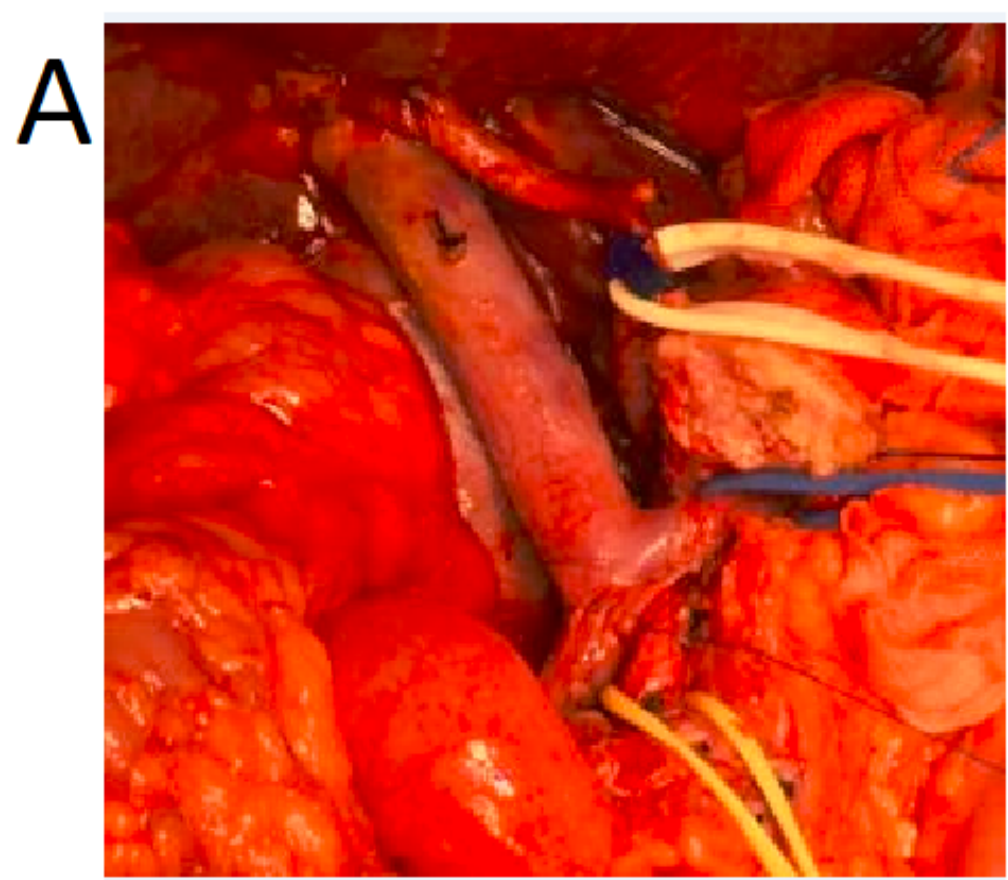
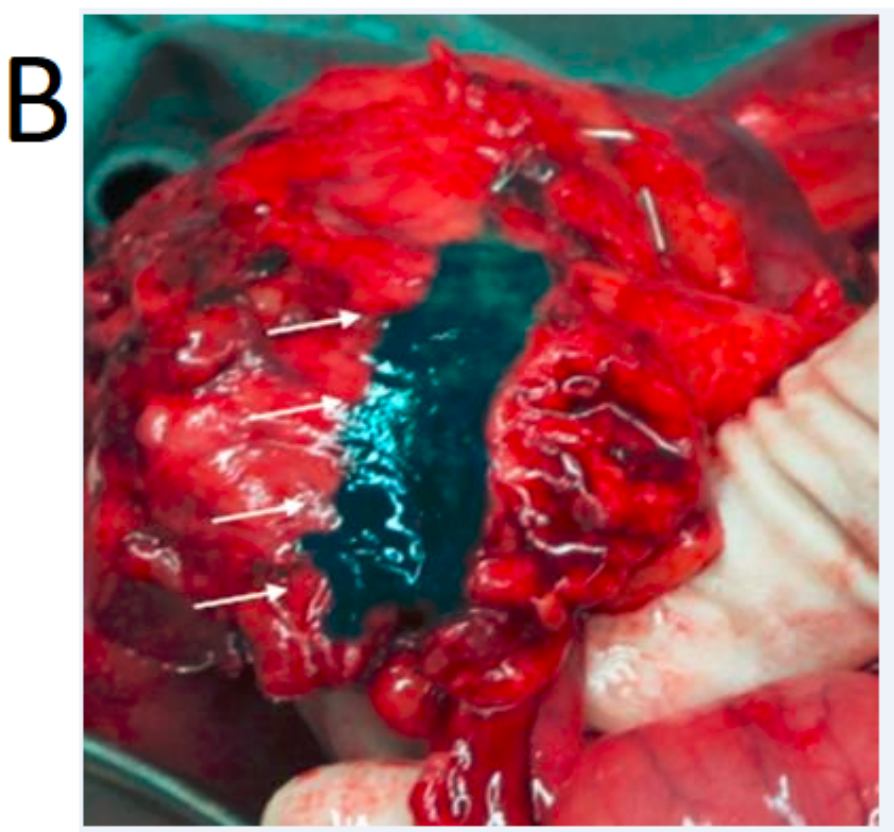

\section{Figure 1}

The resection margin of PV/SMV, SMA: (A) The PV/SMV, SMA surface after radical pancreaticoduodenectomy. (B)Stained the PV/SMV, SMA groove of the pancreaticoduodenectomy resection specimen. 

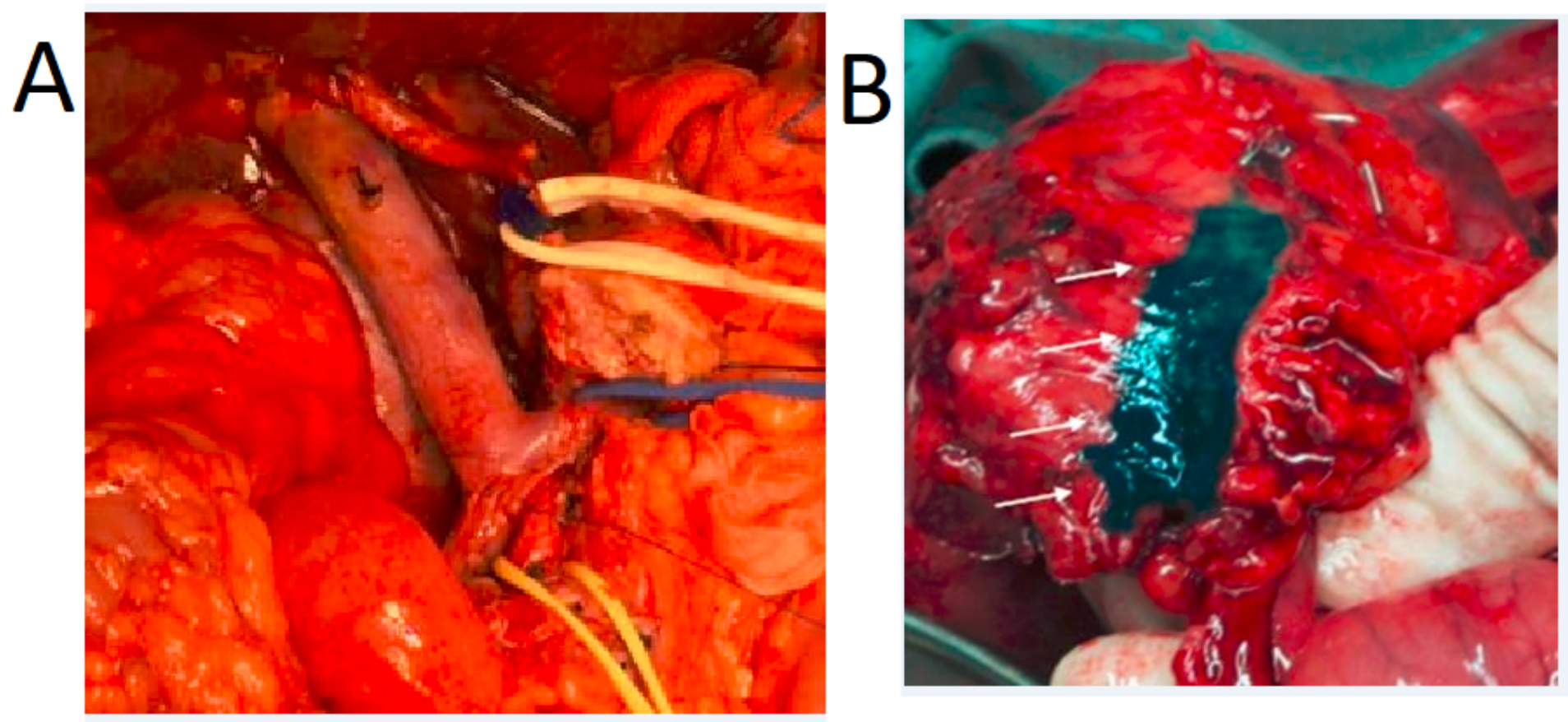

\section{Figure 1}

The resection margin of PV/SMV, SMA: (A) The PV/SMV, SMA surface after radical pancreaticoduodenectomy. (B)Stained the PV/SMV, SMA groove of the pancreaticoduodenectomy resection specimen.

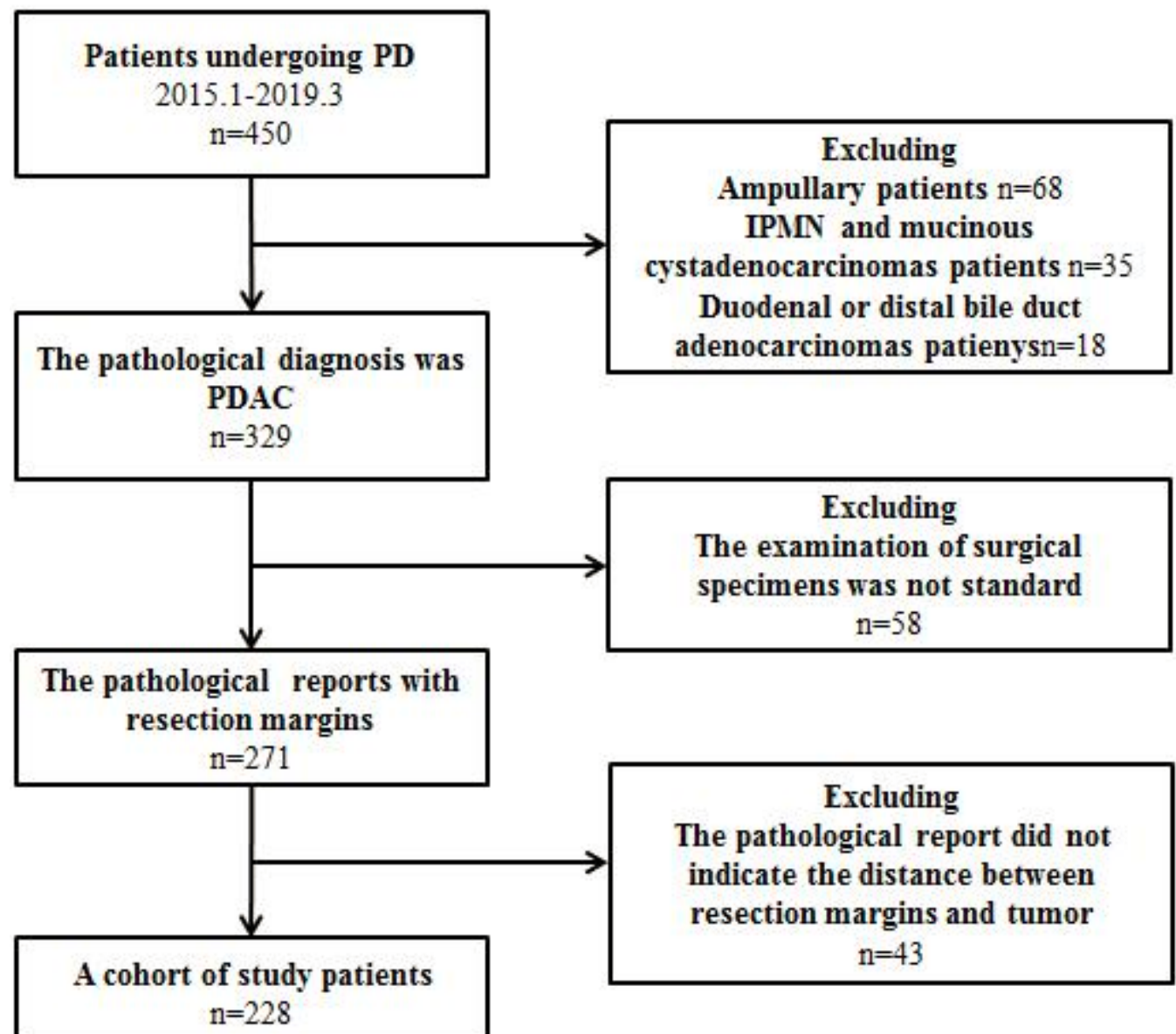


Figure 2

Flow chart of screening patients.

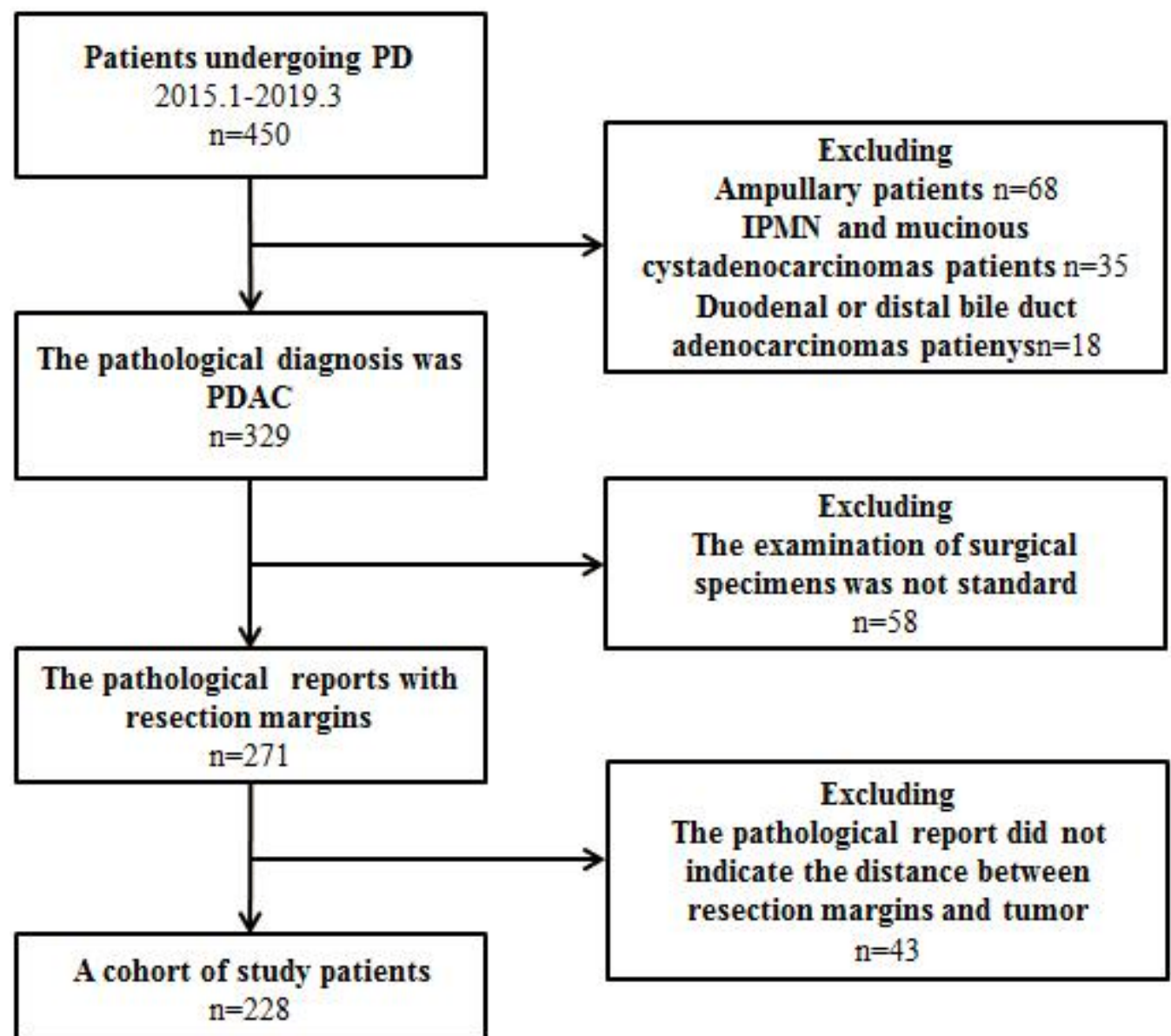

Figure 2

Flow chart of screening patients. 

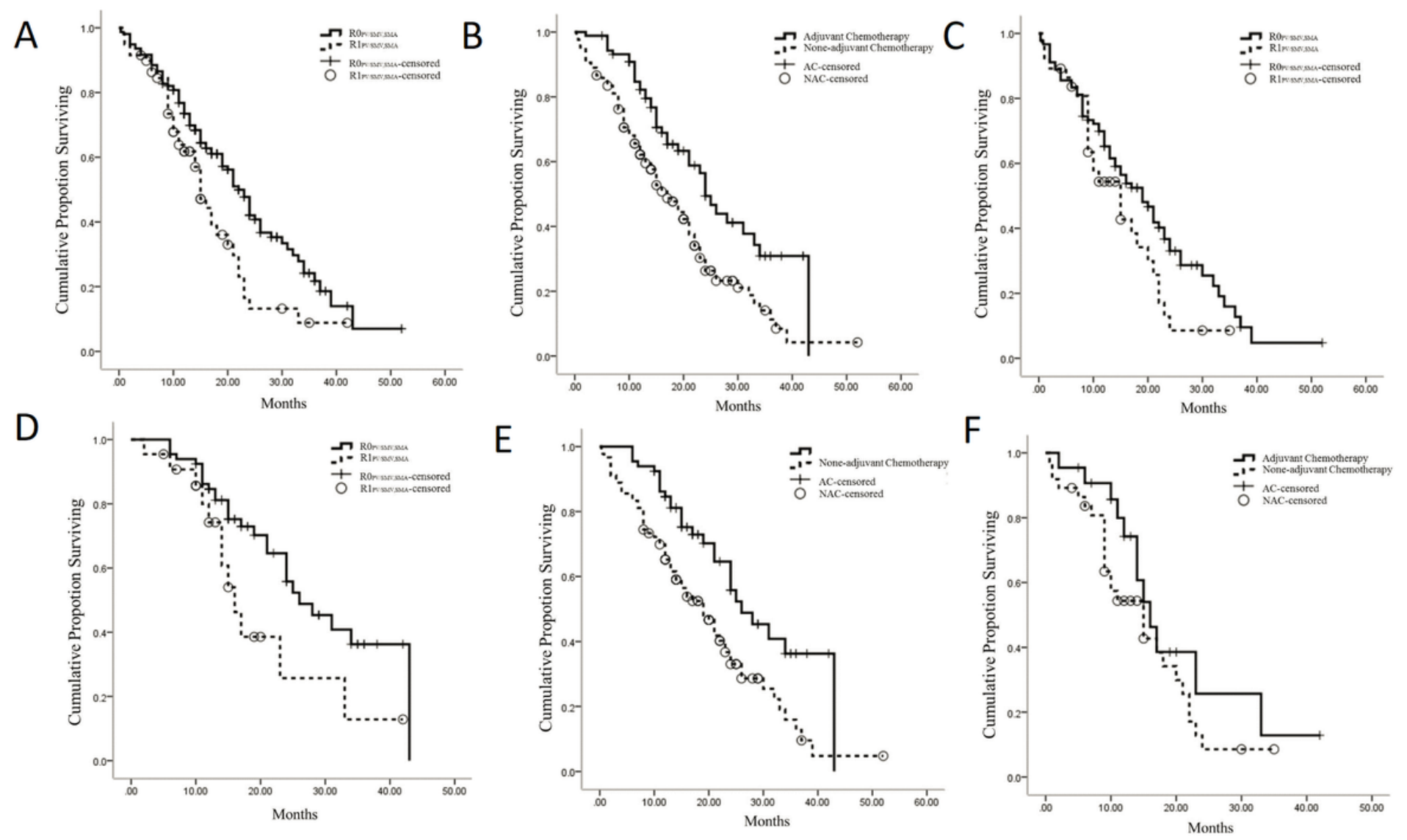

\section{Figure 3}

Kaplan-Meier Survival Curves for the cohort of patients: (A) The median survival time of 166 ROPV/SMV, SMA patients was 22 months compared with 15 months of 62 R1 PV/SMV, SMA patients $(P=0.005)$.(B) The median survival time of 95 adjuvant chemotherapy patients was 24 months compared with 17 months of 133 none-adjuvant chemotherapy patients $(P=0.000)$. (C) In the 133 none-adjuvant chemotherapy patients, the median survival time of 96 ROPV/SMV, SMA patients was 19 months compared with 15 months of 37 R1 PV/SMV, SMA patients $(P=0.074)$. (D) In the 95 adjuvant chemotherapy patients, the median survival time of 70 ROPV/SMV, SMA patients was 26 months compared with 16 months of 25 R1PV/SMV, SMA patients $(P=0.025)$. (E) In the 166 ROPV/SMV, SMA patients, the median survival time of 70 adjuvant chemotherapy patients was 26 months compared with 19 months of 96 none-adjuvant chemotherapy patients $(P=0.001)$. $(F)$ in the $62 R 1 P V / S M V$, SMA patients, the median survival time of 25 adjuvant chemotherapy patients was 16 months compared with 15 months of 37 none-adjuvant chemotherapy patients $(P=0.001)$. 

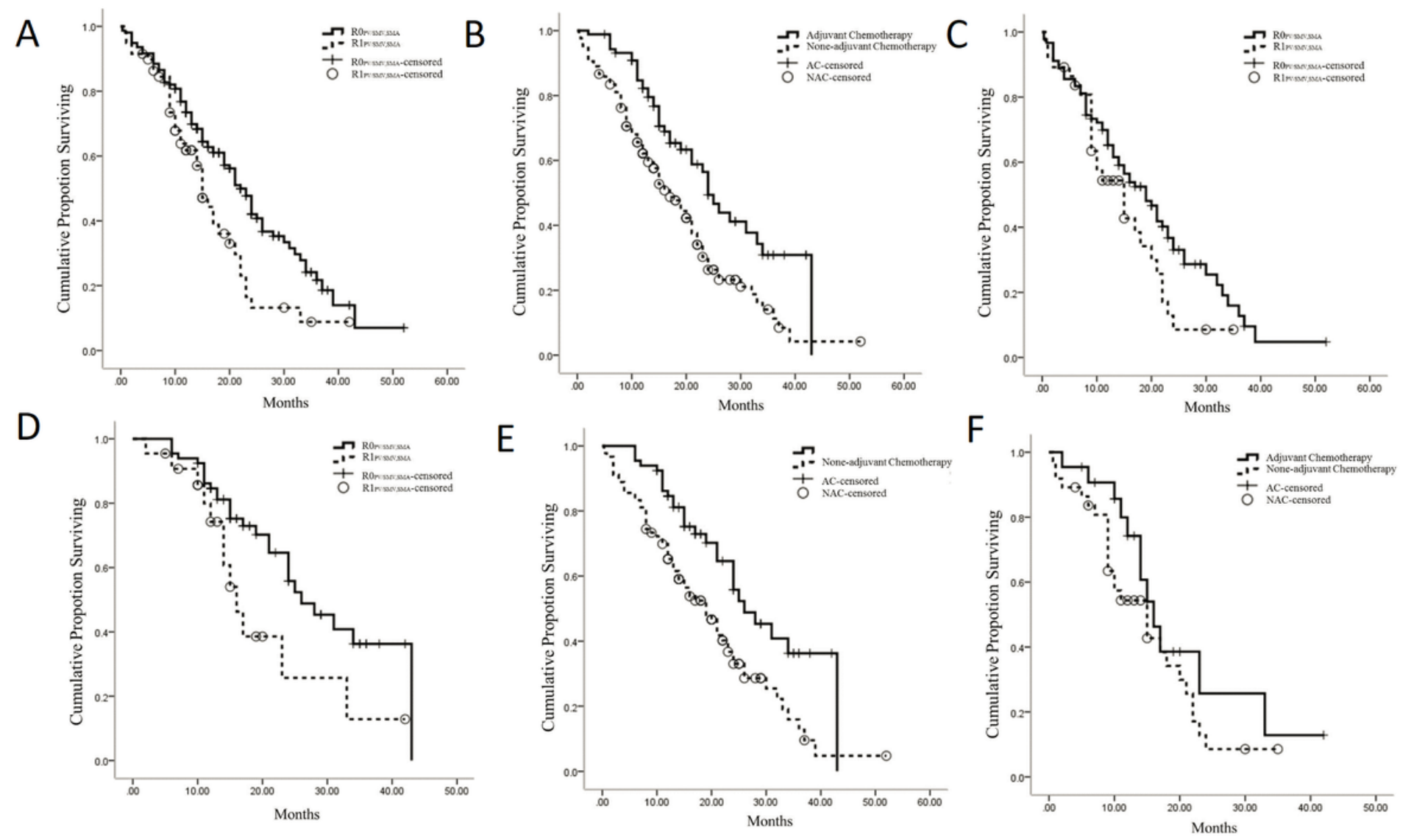

\section{Figure 3}

Kaplan-Meier Survival Curves for the cohort of patients: (A) The median survival time of 166 ROPV/SMV, SMA patients was 22 months compared with 15 months of 62 R1 PV/SMV, SMA patients $(P=0.005)$.(B) The median survival time of 95 adjuvant chemotherapy patients was 24 months compared with 17 months of 133 none-adjuvant chemotherapy patients $(P=0.000)$. (C) In the 133 none-adjuvant chemotherapy patients, the median survival time of 96 ROPV/SMV, SMA patients was 19 months compared with 15 months of 37 R1 PV/SMV, SMA patients $(P=0.074)$. (D) In the 95 adjuvant chemotherapy patients, the median survival time of 70 ROPV/SMV, SMA patients was 26 months compared with 16 months of 25 R1PV/SMV, SMA patients $(P=0.025)$. (E) In the 166 ROPV/SMV, SMA patients, the median survival time of 70 adjuvant chemotherapy patients was 26 months compared with 19 months of 96 none-adjuvant chemotherapy patients $(P=0.001)$. $(F)$ in the $62 R 1 P V / S M V$, SMA patients, the median survival time of 25 adjuvant chemotherapy patients was 16 months compared with 15 months of 37 none-adjuvant chemotherapy patients $(P=0.001)$.

\section{Supplementary Files}

This is a list of supplementary files associated with this preprint. Click to download.

- STROBEchecklistcohort.docx 
- STROBEchecklistcohort.docx

Page 26/26 